\title{
The Ising Model as a Playground for the Study of Wetting and Interface Behavior
}

\author{
D. P. Landau ${ }^{1}$, Alan M. Ferrenberg ${ }^{1,2}$, and K. Binder ${ }^{3}$ \\ ${ }^{1}$ Center for Simulational Physics, The University of Georgia, \\ Athens GA 30603, U.S.A. \\ ${ }^{2}$ University computing and Networking Services, \\ the University of Georgia, Athens, GA 30602 U.S.A. \\ ${ }^{3}$ Institut fuer Physik, Johannes Gutenberg Universitaet Mainz, \\ D-55099 Mainz, Germany
}

Received on 8 December, 2000

\begin{abstract}
Computer simulations have played an important role in the elucidation of wetting and interface unbinding phenomena. In particular, use of the Ising-lattice-gas model in a film geometry and subject to diverse surface and bulk magnetic fields has permitted extensive Monte Carlo simulations to reveal new features of the phase diagrams associated with these phenomena and to provoke new theoretical studies. The status of our knowledge about the nature of wetting and interfacedelocalization transitions which has resulted from these Ising model simulations will be summarized.
\end{abstract}

\section{Introduction}

The study of surface wetting phenomena has a long history with much experimental and theoretical research having been carried out to examine the nature of the resultant behavior. In comparison, computer simulations studies have been rather limited, largely because of the technical difficulties in dealing with realistic models of liquids and liquid-wall interactions. A major advance in this area was made by Nakanishi and Fisher[1] who drew the analogy between wetting phenomena and surface critical behavior in the Ising model. Consequently, a powerful approach to the study of wetting with short range interactions is to use an Ising-lattice-gas model and to take advantage of all of the "technology" which has been developed for performing Monte Carlo simulation of Ising models.

Within the context of the Ising-lattice-gas model we can best examine wetting behavior by considering a semi-infinite Ising model with a simple interaction between "spins" and between "spins" and the walls. The simplest aqpproach to the realization of this geometry on a finite computer is to use a very thick Ising slab for which the top and bottom surfaces are so widely separated that each acts as thoough it is the boundary to a semi-infinite system. On the other hand, thin fluid films that are adsorbed on substrates or confined in slit-like capillaries pose challenging fundamental questions due to a subtle interplay between finite size and surface phenomena. The recognition of this situation has resulted in great activity which has attempted to elucidate various features of these phenomena [2]-[17]. (Such systems can easily be simulated using the same slab geometry described ealier for the study of semiinfinite systems by merely reducing the thicknes of the system being simulated.) Of particular interest is the case where the two surfaces of the fluid film favor different phases: e.g., in the case of a fluid near a gas-liquid coexistence in the bulk, one wall favors high-density liquid and the other wall prefers low-density gas. Similarly, for a binary mixture $(A, B)$ undergoing phase separation in the bulk, one surface favors an $A$-rich phase, the other favors a $B$-rich phase. In the latter case, a situation equivalent to such "competing walls" is also often realized when the mixture is on a substrate and the other surface is "free" (i.e., against air [16]). In the following sections we will review the rich variety of behavior that has been found using Monte Carlo studies of these Ising-lattice-gas models in confined geometries of various kinds with different interactions with the wall $(\mathrm{s})$.

\section{Background}

The generic model for the study of wetting and interface behavior is an Ising (or lattice gas) model, where the local order parameter of the corresponding phase 
transition is a pseudospin variable $s_{i}= \pm 1$ at lattice site $i$. The geometry of the system plays an essential role in the application of the model to problems in wetting and interface formation. A slab geometry is used in which periodic boundary conditions are used in both directions parallel to the surfaces, and the top and bottom have free boundary conditions. A bulk field may be applied, playing the role of a chemical potential difference, or a surface field may act on the surfaces alone. Restricting the spin-spin interactions to nearest neighbor pairwise interactions $J$ in the bulk and $J_{s}$ in the surface planes, we find that the Hamiltonian for this model is

$$
\mathcal{H}=-J \sum_{<i, j>}^{(b)} s_{i} s_{j}-J_{s} \sum_{<i, j>}{ }^{(s)} s_{i} s_{j}-H_{1} \sum_{<i \in n=1>} s_{i}-H_{D} \sum_{<i \in n=D>} s_{i}
$$

where the sum $\sum_{<i, j>}{ }^{(b)}$ runs once over all pairs of neighboring spins where at least one site is not in a surface plane, while the sum $\sum_{<i, j>}{ }^{(s)}$ is limited to pairs with both sites in one of the same (two) surfaces. It is possible to study wetting in this model by examining very thick geometries with identical fields at each surface. The system is initially magnetized in one direction and an applied surface field attempts to overturn spins at the surface. It is important, however, that the thickness of the system is sufficiently great that any overturned layers of spins at the two surfaces are far from each other so that the system appears to be semi-infinite. The schematic wetting phase diagrams predicted by Nakanishi and Fisher[1] are shown in Fig.1 for three different strengths of surface layer coupling $\mathbf{J}_{s}$. These show wetting transitions below $T_{c}(\infty)$ that may be of first order or second order, depending upon the temperature, surface field and surface layer coupling. In addition to the wetting transitions, pre-wetting surfaces are predicted to appear in the presence of a bulk field. For different enhancement of the surface layer coupling the tricritical wetting transition may disappear completely.
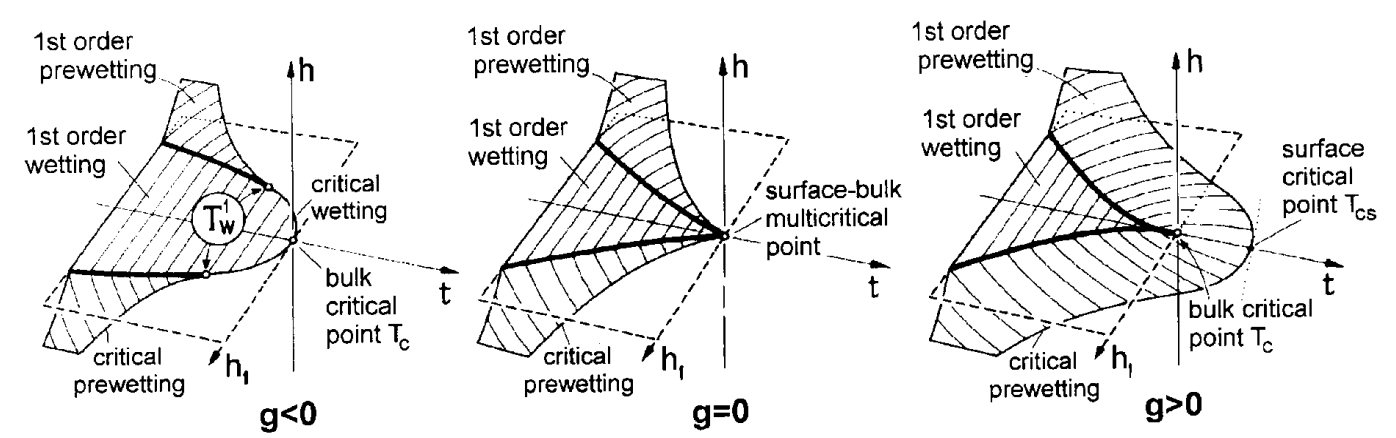

Figure 1. Schematic phase diagrams for wetting transitions in the Ising-lattice gas model. Views as a function of temperature, field and surface field are shown for three different values of the surface exchange enhancement.

A quite different picture is expected for simple interface unbinding studies for which competing surfaces at lattice planes $n=1$ and $n=D$ are described by surface fields of opposite sign, $H_{1}=-H_{D}$. At high temperatures the interface is in the middle so the mean value of $M$, the order parameter, is zero. For temperatures below the critical temperature $T_{c b}$ in the bulk, but sufficiently above the wetting transition $T_{w}$ of the corresponding semi-infinite system $(D \rightarrow \infty)$, this ge- ometry stabilizes a coexistence between two phases of opposite sign of the order parameter, with a freely fluctuating interface in the middle of the film, and mean order parameter is still zero. However, a phase transition appears at $T_{c}(D)[7]-[10],[12]-[14]$ such that for $T<T_{c}(D)$, the interface becomes localized at one of the walls, and then the average order parameter of the film is nonzero. Since $T_{c}(D \rightarrow \infty) \rightarrow T_{w}$ rather than $T_{c b}$, unlike the more familiar situation of non- 
competing walls where "capillary condensation" [6],[11] occurs, this unconventional phase transition has evoked great interest [7]-[10],[12]-[14]. Most work, however, addresses the issue of critical wetting for the corresponding semi-infinite geometry [18],[19], and the transition in a thin film is also 2 nd order [8],[10],[12]-[14]. Even with short range forces, one can obtain pronounced 1st order wetting transitions in semi-infinite geometry by choosing a suitable enhancement of the pairwise interaction near the wall[18] such as in Eq.(1). A mean field theory [9] which treated the corresponding thin film case suggested that 1st order interface localization transitions would be found as the thickness varied.

It is well known that in real fluids the long range of the van der Waals forces exerted by walls on the fluid molecules has the consequence that the wetting transition is almost always 1st order [5] in a semi-infinite geometry. Thus, it is likely that under many circumstances the interface localization-delocalization transition in thin films is 1st order as well. Nonetheless the rich variety of pheonmena uncovered via Monte Carlo simulations could be valuable in describing certain physical situations[16],[20].

\section{Results of Monte Carlo Sim- ulations}

If very thick slabs with equal walls are studied, Monte Carlo simulations[18] reveal wetting transitions that generally substantiate the picture envisoned by Nakanishi and Fisher. If the slab is magnetized up and a surface field is applied in the opposite direction, a well defined interface forms between a layer of overturned spins at the surface and the magnetized bulk. (Far from the surface the magnetization reaches the value that it has in a system with periodic boundaries and is essentially that for an infinite system.) Profiles, such as those in Fig.2, show that the interface moves away from the surface as the magnitude of the surface field $H_{1}$ increases. Furthermore, these profiles show that even when the interface is far from the surface there is a small variation of the magnetization in the vicinity of the surface that is described by a length scale that is distinct from the distance from the interface to the wall. At low temperatures the transition is 1st order, and as the temperature is increased the magnitude of the discontinuity at the transition decreases until a tri- critical point is reached beyond which the transition is 2nd order. This behavior, complete with hysteresis for the first order transition, is demonstrated in the insert to Fig.2. At very low temperatures, i.e., below the roughening transition, layering occurs; and as the surface field is varied, the thickness of the film increases one layer at a time instead of via a single wetting transition. We will not discuss this layering further except to say that there are still interesting questions to be answered regarding the manner in which the layering gives way to wetting.

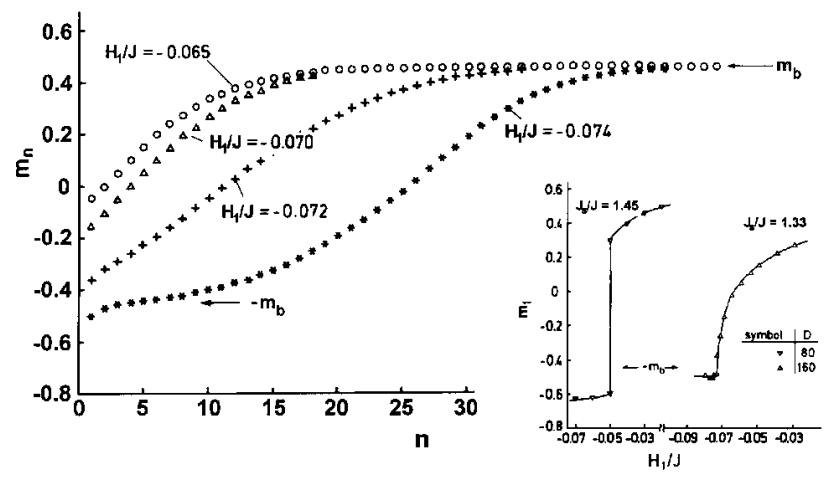

Figure 2. Profiles of magnetization in a thick Ising film as a function of distance from the surface for a $128 \times 128 \times 160$ with $J_{s} / J=1.33$.. The inset shows the variation of the surface layer magnetization with surface field for two different values of enhanced surface coupling.

The nature of the critical behavior associated with the 2 nd order wetting transition had been predicted by a renormalization group treatment[21] to be nonuniversal. The quantities that enter the dimensionless parameter that was expected to control the critical behavior were all measureable for the Ising model so a firm prediction was possible. Surprisingly, when data from simulations on systems covering a wide range of sizes were carefully analyzed for both the surface layer magnetization and the surface layer susceptibility, the conclusion was that the critical behavior was indeed mean field like! This finding inspired a number of new theoretical studies culminating in the introduction, by Boulter and Parry[23], of a second length into the theory that described the variation of the magnetization in the vicinity of the wall with the result that the theoretical prediction was modified. Although we do not yet have a final resolution, it is clear that in this case simulation was instrumental in encouraging additional theoretical work with substantial new physics resulting from the effort. 


\section{competition: surface phenomena $\Leftrightarrow$ finite size effects}

\section{both walls attractive}

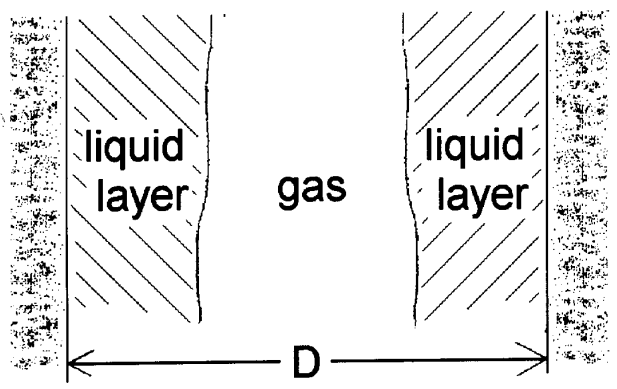

\section{one wall repulsive, one wall attractive}

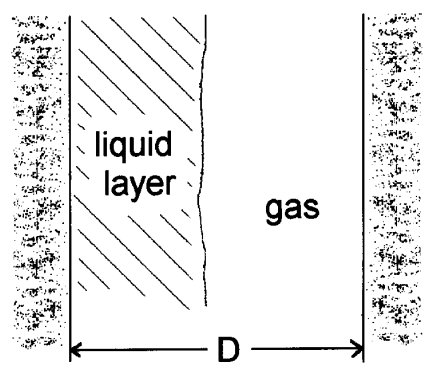

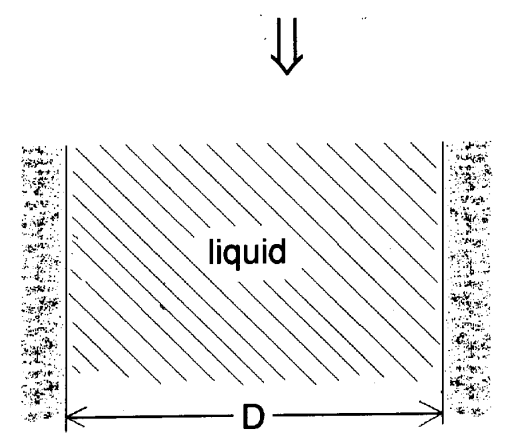

Capillary condensation
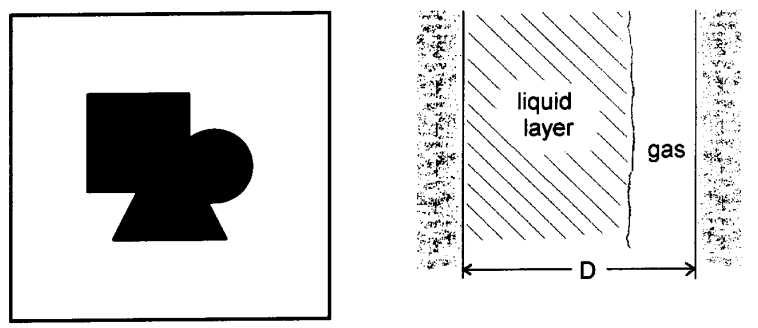

\section{Interface delocalization}

Figure 3. Schematic view of the comparison between capillary condensation and interface delocatization in a thin slit geometry.

Wetting behavior in thin capillaries is quite different from the scenario just described for thick systems, and a schematic view of the two possibile kinds of characteristic behavior is shown in Fig.3. In thin capillaries with equal walls the entire volume fills up with fluid more easily than wetting would occur in the semi-infinite system, and capillary condensation results. Monte Carlo simulations[22] showed that the qualitative features of the phase diagram remain similar to that for the bulk, but the entire boundary is shifted in chemical potentialfield space. The resultant phase diagram, shown in Fig. 4, has no trace of any unusual behavior at the wetting transition in thick systems, but the critical temperature is depressed relative to that in the infinite system. This means, of course, that the density-temperature (i.e., magnetization-temperature) phase diagram also acquires an asymmetry, and the critical point is shifted away from $50 \%$ density. Thus, in the end, the capillary condensation in thin slits is relatively straightforward to understand.
In thin capillaries with opposite walls the Isinglattice-gas model provides a laboratory for the study of interesting interface behavior. In this geometry the phase transition occuring in the bulk at a temperature $T_{c b}$ is supressed and instead an interface forms between coexisting phases that are determined by the surface fields. Typical profiles, such as those shown in Fig.5 for $L=12$, reveal that above the transition when the interface is in the center of the film, the overall magnetization is zero. At a temperature $T_{c}(D)<T_{c b}$ the interface becomes bound to one wall or the other and an interface localization transition takes place. The results of the simulations show that the transition is shifted below the wetting transition in a semi-infinite system and belongs to the universality class of the two dimensional Ising model. In addition, between this transition and $T_{c b}$ the maximum value of the layer susceptibility varies exponentially with the thickness $D$ of the film and is governed by a new length $l$ that characterizes the interface. In contradiction to theoretical 
expectations[5], $l$ is different than the bulk correlation length.

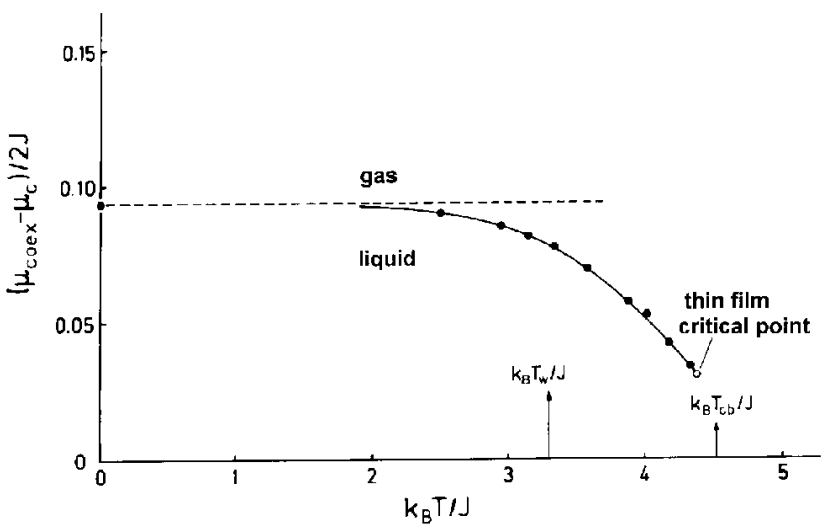

Figure 4. Phase boundary for capillary condensation in a capillary of thickness $D=16$ and wall potential $H_{1}=$ -0.75 . The arrows show the location of the wetting transition in the bulk $T_{w}$ and the bulk critical temperature $T_{c b}$.

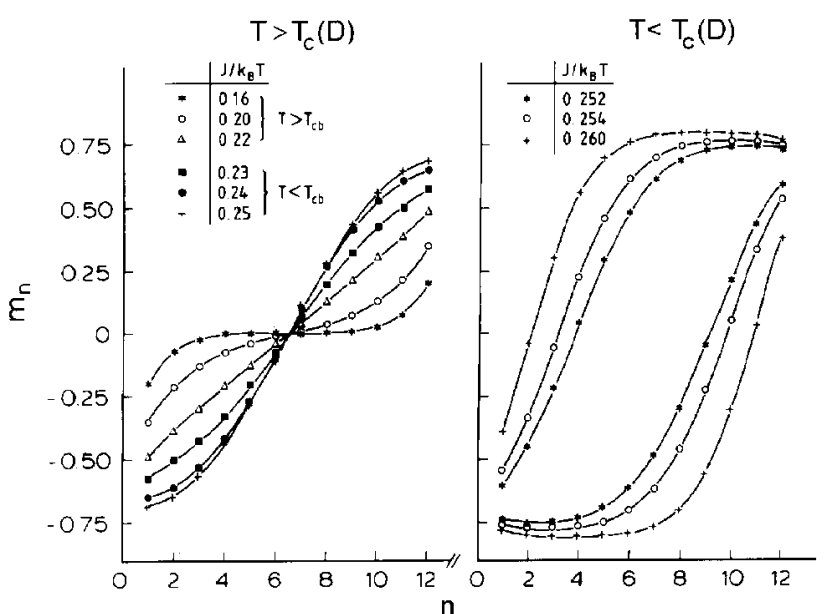

Figure 5. Profiles of the layer magnetization plotted vs layer number for a thin film $(\mathrm{L}=12)$ with unlike walls. On the left are profiles for $T>T_{c}(D)$, and on the right are profiles for $T<T_{c}(D)$ with the system initially magnetized with all spins up or all spins down. Curves are only a guide to the eye.

To add still further to the range of intriguing behavior that can occur when the capillary has unlike walls, we turn to the case when the surface coupling is enhanced. Then, as the thickness of the capillary is modified, the transition can change from 1st order to 2nd order[29]. Using Monte Carlo simulations with well over $10^{6}$ MCS per data point we examined the behavior for several values of $J_{s} \geq 1.3 J$, where the wetting transition at the surface of a semi-infinite system would be very strongly first order at $J / k_{B} T=0.25$. In a thin film we found that the tricritical point, i.e., the ratio $J_{s t} / J$ where the transition changes from 2 nd order to 1st order, is enhanced. This also implies that at a fixed ratio of $J_{s} / J$ there might be a 2 nd order transition for thin films and a 1st order transition for thick films; i.e., the order of the interface localizationdelocalization transition can be modified by changing the thickness of the film. Thus, a tricritical point occurs at fixed $J_{s} / J$ at some critical thickness $D_{t}$. Simulations were limited to very thin films $(D=4,6,8)$ since for thick films, slow interfacial fluctuations made runs impossibly long. Multiple quantities, such as the average order parameter $\langle|M|>$ and the logarithmic derivative of $\left\langle|M|>\right.$, were examined. For $J_{s} / J=1.3$ and $D=6$ the variation of the order parameter near $K_{c}=J / k_{B} T_{c}$ is very smooth and strongly rounded by finite size: The data have exactly the same features as corresponding data for $J_{s} / J=1$ [13],[14] and all related evidence [26] (e.g. profiles of order parameter, energy etc. across the film) support the conclusion that the transition is still 2nd order (although it is 1st order for $D \rightarrow \infty[18]$ ). For $J_{s} / J=1.5$, however, a steep variation of $\langle|M|\rangle$ with $K$ indicates that this is already a rounded 1st order transition. This interpretation is supported by the positions of the maxima of other quantities that have characteristic divergencies at the transition. Monte Carlo data also showed that the tricritical point could also be reached by varying the thickness $D$ while holding the surface coupling constant. (This approach is more likely to be realized in an experiment.) For example, a finite size scaling analysis of the data for the logarithmic derivative of $|M|$ shows that the positions of the peaks are compatible with the characteristic behavior $K_{\max }(L)-K_{c} \propto L^{-2}$ for 1st order transitions [27] while in the 2nd order case $K_{\max }(L)-K_{c} \propto L^{-1}[13],[14]$ (at a 2 nd order transition the extrapolation should vary as $L^{-1 / \nu}$, where $\nu=1$ for the 2-d Ising-like delocalization transition). Lee and Kosterlitz' [28] analysis of the free energy barrier to determine the order of a phase transition produces the same result. For $J_{s} / J=1.45$ the energy distribution for $D=4$ shows only a single peak for all lattice sizes (i.e., the transition is 2 nd order), but for $D=8$ the distribution is double peaked and the resulting free energy barrier increases rapidly with $L$ (i.e., the transition is strongly 1st order). Additional strong evidence for the change of the order of the transition comes from a study of the variation of the maxima of the specific heat, susceptibility, as well as the logarithmic derivative of $|M|$ with $\mathrm{L}$.

\section{Conclusion}

In conclusion, we have presented evidence that the Ising-lattice gas model provides the opportunity to investigate a rich variety of behavior involving wetting and interfaces. Data for thick films revealed the sur- 
prising result that critical wetting is mean-field in nature, in contradiction to the prevailing theory. This simulational work prompted further theoretical effort which revised the prevailing view by observing that a potentially critical element had been left out of the theory. In thin Ising films with opposing walls one can change the order of the transition from 2 nd to 1 st order by increasing the thickness, keeping the surface fields and exchange couplings near the wall constant. The occurence of such a tricritical point can be inferred from the mean field treatment of Swift et al [9], but Monte Carlo simulations provide evidence that this new type of tricritical point persists beyond mean field theory. The critical behavior of this special tricritical point still remains to be investigated [26]. It is also interesting to ask which features of our results will carry over to real systems. For liquid-gas transitions, one expects that the van der Waals forces imply first order wetting [5], and it is not clear whether second order interface localization transitions become possible in a thin film geometry. On the other hand, for "symmetrical" binary polymer mixtures it is conceivable that the difference between the van der Waals forces of the two species is very small, and an effectively short-range interaction dominates [16]. Recent experiments on interfaces in confined geometry are consistent with such a picture, but experimental evidence for an interface localization transition is still lacking. Clearly, more experiments on related systems are needed to resolve these issues.

Acknowledgements: This research was supported in part by NSF grant DMR-9727714 and DFG grant SFB262/D1.

\section{References}

[1] H. Nakanishi and M.E. Fisher, J. Chem. Phys. 78, 3279 (1983)

[2] Fluid Interfacial Phenomena, edited by C.A. Croxton (Wiley, New York, 1986).; Liquids at Interfaces, edited by J. Charvolin, J.F. Joanny, and J. Zinn-Justin (NorthHolland, Amsterdam, 1990).

[3] Physics of Polumer Surfaces and Interfaces, edited by I.C. Sanchez (Butterworth-Heinemann, Boston, 1992).

[4] K. Binder, in Phase Transitions and Critical Phenomena, edited by C. Domb and J.L. Lebowitz (Academic, London, 1983), Vol. 8, p. 1.

[5] S. Dietrich, in Phase Transitions and Critical Phenomena, edited by C. Domb and J.L. Lebowitz (Academic, London, 1988), Vol. 11, p. 1.

[6] H. Nakanishi and M.E. Fisher, J. Chem. Phys. 78, 3279 (1983).

[7] E.V. Albano, K. Binder, D.W. Heermann, and W. Paul, Surf. Sci. 223, 157 (1989).
[8] A.O. Parry and R. Evans, Phys. Rev. Lett. 64, 439 (1990).

[9] M.R. Swift, A.L. Owczarek, and J.O. Indekeu, Europhys. Lett. 14, 475 (1991).

[10] A.O. Parry and R. Evans, Physica A 181, 250 (1992).

[11] K. Binder and D.P. Landau, J. Chem. Phys. 96, 1444 (1992).

[12] K. Binder, A.M. Ferrenberg and D.P. Landau, Ber. Bunsenges. Phys. Chemie 98, 340 (1994).

[13] K. Binder, D.P. Landau, and A.M. Ferrenberg, Phys. Rev. Lett. 74, 298 (1995); Phys. Rev. E 51, 2823 (1995).

[14] K. Binder, R. Evans, D.P. Landau, and A.M. Ferrenberg, Phys. Rev. E 53, 5023 (1996).

[15] Y. Rouault, J. Baschnagel, and K. Binder, J. Stat. Phys. 80, 1009 (1995).

[16] T. Kerle, J. Klein and K. Binder, Phys. Rev. Lett. 77, 1318 (1996)

[17] A. Werner, F. Schmid, K. Binder and Mueller, J. Chem. Phys. 107, 8175 (1997).

[18] K. Binder and D.P. Landau, Phys. Rev. B 37, 1745 (1988); K. Binder, D.P. Landau and S. Wansleben, Phys. Rev. B 40, 6971 (1989).

[19] It has been suggested [M.E. Fisher and A.J. Jin, Phys. Rev. Lett. 69, 792 (1992)] that the wetting transition of the Ising model is never second order but actually weakly first order for $J_{s}=J$. If this is true, it would only affect the behavior extremely close to $T_{c}(D)$, a region that is not accessible here.

[20] T. Kerle, J. Klein, and K. Binder, Euro. Phys. J. B 7, 401 (1999); D. Ross, D. Bonn, and J. Meunier (to be published).

[21] E. Brezin, B. I. Halperin, and S. Leibler, Phys. Rev. Lett.50, 1387 (1983); R. Lipowsky, D. M. Kroll, and R. K. P. Zia, Phys Rev. B 27, 4499 (1983); D. S. Fisher and D. A. Huse, Phys. Rev. B 32, 247 (1985); R. Lipowsky and M. E. Fisher, Phys. Rev. B 36, 2126 (1987).

[22] K. Binder and D. P. Landau, J. Chem. Phys. 96, 1444 (1992).

[23] C. J. Boulter and A. O. Parry, Phys. Rev. Lett 74, 3403 (1995); Physica A 218109 (1995): A. O. Parry and C. J. Boulter, Physica A 218, 77 (1995).

[24] A.M. Ferrenberg and R.H. Swendsen, Phys. Rev. Lett. 61, 2635 (1988); 63, 1195 (1989).

[25] A.M. Ferrenberg, D.P. Landau and R.H. Swendsen, Phys. Rev. E 51, 5092 (1995).

[26] See: K. Binder, Rep. Prog. Phys. 60, 487 (1997), and references therein.

[27] D. P. Landau, in Finite Size Scaling and Numerical Simulations of Statistical Systems (World Scientific, Singapore, 1990).

[28] J. Lee and J.M. Kosterlitz, Phys. Rev. Lett. 65, 137 (1990).

[29] A. M. Ferrenberg, D. P. Landau, and K. Binder, Phys. Rev. B 58, 3353 (1998). 